\title{
A Study of Psychosocial Factor Affecting Attitude toward Suicide of Adult Male and Female Inhabiting in a Province of Korea
}

\author{
Sang-Yeol Lee, Hye-Jin Lee
}

Department of Psychiatry, Wonkwang University School of Medicine, Republic of Korea

Department of Public Health, Wonkwang Graduate School, Republic of Korea

Background: Individual attitudes toward suicide are known to markedly influence suicidal ideation and suicidal behavior. Therefore, this study aimed to determine the factors associated with attitudes toward suicide.

Methods: This study was conducted on 3,213 adult men and women living in a province of Korea. After excluding 281 participants because of unreliable responses, the remaining 2,932 were included in the data analysis. Demographic factors were investigated, and psychological factors were evaluated. Attitudes toward suicide were evaluated using the Attitudes Towards Suicide-20, physical symptoms were evaluated using the Patient Health Questionnaire-15 (PHQ-15), self-compassion was evaluated using the Korean Self-Compassion Scale, and stress was evaluated using the Global Assessment of Recent Stress (GARS) scale. One-way analysis of variance was used to examine the psychological characteristics of attitudes toward suicide.

Conclusion: This study explored the demographic and psychological factors affecting attitudes toward suicide. Adequate mediation of these factors is needed

Table 2. Comparisons suicidal ideation, suicidal plan, and suicide attempt among subgroups of attitude towards suicide

\begin{tabular}{|c|c|c|c|c|c|c|}
\hline Variable & & $\mathrm{N}(\%)$ & Factor 1 & Factor 2 & Factor 3 & Factor 4 \\
\hline \multirow{7}{*}{$\begin{array}{l}\text { Suicidal idea } \\
\text { tion }\end{array}$} & None (a) & $\begin{array}{c}2124(72.4 \\
)\end{array}$ & $\begin{array}{c}19.23 \pm 5 . \\
30\end{array}$ & $\begin{array}{c}13.53 \pm 3 . \\
15\end{array}$ & $\begin{array}{c}10.93 \pm 2 . \\
74\end{array}$ & $\begin{array}{c}11.93 \pm 2 . \\
97\end{array}$ \\
\hline & $\begin{array}{l}<1 \text { year }(\mathrm{b} \\
\text { ) }\end{array}$ & 278(9.5) & $\begin{array}{c}21.82 \pm 5 . \\
64\end{array}$ & $\begin{array}{c}14.07 \pm 2 . \\
90\end{array}$ & $\begin{array}{c}11.73 \pm 2 . \\
97\end{array}$ & $\begin{array}{c}11.81 \pm 2 . \\
88\end{array}$ \\
\hline & $\begin{array}{l}>1 \text { year }(\mathrm{c} \\
\text { ) }\end{array}$ & 530(18.1) & $\begin{array}{c}20.57 \pm 5 . \\
11\end{array}$ & $\begin{array}{c}14.41 \pm 2 . \\
74\end{array}$ & $\begin{array}{c}11.14 \pm 2 . \\
72 .\end{array}$ & $\begin{array}{c}11.60 \pm 2 . \\
85\end{array}$ \\
\hline & $\mathrm{F} / \mathrm{p}$ value & & $F=37.73$ & $F=19.50$ & $F=10.77$ & \\
\hline & Post-hoc & & 0 & 2 & 3 & $F=2.715$ \\
\hline & (Bonferon & & $p<0.001$ & $p<0.001$ & $p<0.001$ & $P=0.066$ \\
\hline & ni) & & $b>c>a$ & $\mathrm{~b}, \mathrm{c}>\mathrm{a}$ & $\mathrm{b}>\mathrm{a}, \mathrm{c}$ & \\
\hline \multirow{6}{*}{ Suicidal plan } & None (a) & $\begin{array}{c}2605(88.8 \\
)\end{array}$ & $\begin{array}{c}19.45 \pm 5 . \\
28\end{array}$ & $\begin{array}{c}13.71 \pm 3 . \\
08\end{array}$ & $\begin{array}{c}11.00 \pm 2 . \\
73\end{array}$ & $\begin{array}{c}11.93 \pm 2 . \\
96\end{array}$ \\
\hline & $\begin{array}{l}<1 \text { year }(\mathrm{b} \\
\text { ) }\end{array}$ & 104(3.6) & $\begin{array}{c}22.56 \pm 5 . \\
55\end{array}$ & $\begin{array}{c}13.62 \pm 2 . \\
98\end{array}$ & $\begin{array}{c}12.19 \pm 3 . \\
14\end{array}$ & $\begin{array}{c}12.03 \pm 2 . \\
89\end{array}$ \\
\hline & $>1$ year $(\mathrm{c}$ & 223(7.6) & $\begin{array}{c}21.55 \pm 5 . \\
57\end{array}$ & $\begin{array}{c}14.10 \pm 3 . \\
11\end{array}$ & $\begin{array}{c}11.09 \pm 2 . \\
86\end{array}$ & $\begin{array}{c}10.96 \pm 2 . \\
67\end{array}$ \\
\hline & $\mathrm{F} / \mathrm{p}$ value & & $F=31.43$ & & & $F=11.36$ \\
\hline & Post-hoc & & 2 & $F=1.692$ & $F=9.453$ & 7 \\
\hline & $\begin{array}{l}\text { (Bonferon } \\
\text { ni) }\end{array}$ & & $\begin{array}{c}p<0.001 \\
\mathrm{~b}, \mathrm{c}>\mathrm{a}\end{array}$ & $p=0.184$ & $\begin{array}{l}p<\mathrm{a}, \mathrm{c} \\
p<.001\end{array}$ & $\begin{array}{c}p<0.001 \\
\mathrm{~b}>\mathrm{a}>\mathrm{c}\end{array}$ \\
\hline \multirow{5}{*}{$\begin{array}{l}\text { Suicide atte } \\
\text { mpt }\end{array}$} & None (a) & $\begin{array}{c}2789(95.2 \\
)\end{array}$ & $\begin{array}{c}19.95 \pm 5 . \\
33\end{array}$ & $\begin{array}{c}14.63 \pm 2 . \\
61\end{array}$ & $\begin{array}{c}11.42 \pm 2 . \\
73\end{array}$ & $\begin{array}{c}11.87 \pm 2 . \\
95\end{array}$ \\
\hline & $\begin{array}{l}<1 \text { year }(b \\
\text { ) }\end{array}$ & $36(1.2)$ & $\begin{array}{c}22.67 \pm 4 . \\
84\end{array}$ & $\begin{array}{c}12.25 \pm 4 . \\
18\end{array}$ & $\begin{array}{c}11.78 \pm 4 . \\
15\end{array}$ & $\begin{array}{c}11.42 \pm 3 . \\
99\end{array}$ \\
\hline & $\begin{array}{l}>1 \text { year (c } \\
\text { ) } \\
\text { F/p value }\end{array}$ & 107(3.6) & $\begin{array}{c}20.89 \pm 7 . \\
01 \\
F=18.04\end{array}$ & $\begin{array}{c}13.73 \pm 3 . \\
07\end{array}$ & $\begin{array}{c}11.60 \pm 2 . \\
99\end{array}$ & $\begin{array}{c}11.62 \pm 2 . \\
47\end{array}$ \\
\hline & Post-hoc & & 0 & $F=8.738$ & $F=1.574$ & $F=0.797$ \\
\hline & (Bonferon & & $p<0.001$ & $\begin{array}{l}p<0.001 \\
\mathrm{a}>\mathrm{c}>\mathrm{b}\end{array}$ & $p=0.058$ & $p=0.451$ \\
\hline
\end{tabular}

Table 3. Comparisons factors of attitude towards suicide among groups according to the severity of physical symptoms

\begin{tabular}{|c|c|c|c|c|c|}
\hline ATTS-20 & $\begin{array}{l}\text { Mild (a) } \\
(\mathrm{n}=1653)\end{array}$ & $\begin{array}{c}\text { Moderate (b) } \\
(\mathrm{n}=776)\end{array}$ & $\begin{array}{c}\text { Severe }(c) \\
(\mathrm{n}=503)\end{array}$ & $\mathrm{F} / \mathrm{p}$ value & $\begin{array}{l}\text { Post-hoc } \\
\text { (Bonferonni) }\end{array}$ \\
\hline Factor 1 & $19.26 \pm 5.34$ & $19.88 \pm 5.36$ & $20.98 \pm 5.27$ & $\begin{array}{r}F=20.332 \\
p \ll 0.001\end{array}$ & $a<b<c$ \\
\hline Factor 2 & $14.01 \pm 2.74$ & $13.55 \pm 3.24$ & $13.96 \pm 2.97$ & $\begin{array}{l}F=7.700 \\
p \ll 0.001\end{array}$ & $a<b, c$ \\
\hline Factor 3 & $10.81 \pm 2.79$ & $11.29 \pm 2.67$ & $11.43 \pm 2.77$ & $\begin{array}{r}F=13.741 \\
p \ll 0.001\end{array}$ & $a<b, c$ \\
\hline Factor 4 & $11.81 \pm 3.03$ & $11.92 \pm 2.83$ & $11.92 \pm 2.83$ & $\begin{array}{l}F=0.530 \\
p=0.589\end{array}$ & n.s. \\
\hline
\end{tabular}

Table 1. Comparisons demographic characteristics among subgroups of attitudes towards suicide

\begin{tabular}{|c|c|c|c|c|c|c|}
\hline Variable & & $\mathrm{N}(\%)$ & Factor 1 & Factor 2 & Factor 3 & Factor 4 \\
\hline \multirow{3}{*}{ Sex } & $\begin{array}{l}\text { Male } \\
\end{array}$ & $\begin{array}{l}1408(48.0) \\
\end{array}$ & $19.95 \pm 5.49$ & $13.47 \pm 3.18$ & $11.21 \pm 2.74$ & $11.75 \pm 3.01$ \\
\hline & Female & $1524(52.0)$ & $19.51 \pm 5.24$ & $13.99 \pm 2.96$ & $10.87 \pm 2.79$ & $11.96 \pm 2.87$ \\
\hline & $t / p$ value & & $\begin{array}{r}t=2.235 \\
p=0.026\end{array}$ & $\begin{array}{r}t=-4.606 \\
p<0.001\end{array}$ & $\begin{array}{c}t=3.352 \\
p<0.001\end{array}$ & $\begin{array}{l}t=-1.875 \\
p=0.061\end{array}$ \\
\hline \multirow{6}{*}{$\begin{array}{l}\text { Age } \\
\text { (years) }\end{array}$} & $20 \mathrm{~s}$ (a) & $580(19.8)$ & $20.58 \pm 5.24$ & $14.28 \pm 2.74$ & $10.77 \pm 2.62$ & $11.44 \pm 2.76$ \\
\hline & $30 \mathrm{~s}(\mathrm{~b})$ & $553(18.9)$ & $18.98 \pm 5.42$ & $14.25 \pm 3.07$ & $10.58 \pm 2.69$ & $11.75 \pm 2.84$ \\
\hline & 40s (c) & $567(19.3)$ & $19.45 \pm 5.24$ & $14.04 \pm 3.07$ & $11.11 \pm 2.86$ & $11.75 \pm 2.99$ \\
\hline & $50 \mathrm{~s}(\mathrm{~d})$ & $542(18.5)$ & $19.65 \pm 5.52$ & $13.43 \pm 3.15$ & $11.40 \pm 2.71$ & $12.08 \pm 2.99$ \\
\hline & Above 60s (e) & $690(23.5)$ & $19.87 \pm 5.32$ & $12.88 \pm 3.09$ & $11.32 \pm 2.86$ & $12.21 \pm 3.05$ \\
\hline & $\begin{array}{l}\text { F/p value } \\
\text { Post-hoc } \\
\text { (Bonferonni) }\end{array}$ & & $\begin{array}{c}F=6.897 \\
p<0.001 \\
\mathrm{e}>\mathrm{b}, \mathrm{a}>\mathrm{b}, \mathrm{c}, \mathrm{d}\end{array}$ & $\begin{array}{c}F=25.177 \\
p<0.001 \\
\mathrm{a}, \mathrm{b}, \mathrm{c},>\mathrm{d}>\mathrm{e}\end{array}$ & $\begin{array}{l}F=9.573 \\
p<0.001 \\
\mathrm{c}, \mathrm{d}, \mathrm{e}>\mathrm{b}, \\
\mathrm{d}, \mathrm{e}>\mathrm{a}\end{array}$ & $\begin{array}{c}F=6.557 \\
p<0.001 \\
\mathrm{~d}, \mathrm{e}>\mathrm{a}\end{array}$ \\
\hline \multirow{6}{*}{$\begin{array}{l}\text { Education } \\
\text { (years) }\end{array}$} & $<6$ (a) & $301(10.3)$ & $20.41 \pm 5.22$ & $12.58 \pm 3.29$ & $11.43 \pm 2.93$ & $11.80 \pm 3.13$ \\
\hline & $7-9$ (b) & 185(6.3) & $20.36 \pm 5.22$ & $12.62 \pm 3.26$ & $11.42 \pm 2.86$ & $12.10 \pm 3.00$ \\
\hline & $10-12$ (c) & $809(27.6)$ & $19.83 \pm 5.57$ & $13.27 \pm 3.21$ & $11.16 \pm 2.85$ & $12.01 \pm 3.02$ \\
\hline & $13-16$ (d) & $1365(46.5)$ & $19.76 \pm 5.29$ & $14.29 \pm 2.81$ & $10.91 \pm 2.65$ & $11.82 \pm 2.82$ \\
\hline & Above 16 (e) & 272(9.3) & $17.99 \pm 5.08$ & $14.43 \pm 2.74$ & $10.71 \pm 2.77$ & $11.48 \pm 3.06$ \\
\hline & $\begin{array}{l}\text { F/p value } \\
\text { Post-hoc } \\
\text { (Bonferonni) }\end{array}$ & & $\begin{array}{l}F=9.199 \\
p<0.001 \\
\mathrm{a}, \mathrm{b}, \mathrm{c}, \mathrm{d} \gg \mathrm{e}\end{array}$ & $\begin{array}{c}F=37.787 \\
p<0.001 \\
\mathrm{~d}, \mathrm{e}>\mathrm{a}, \mathrm{b}, \mathrm{c} \\
\mathrm{c}>\mathrm{a}\end{array}$ & $\begin{array}{c}F=4.510 \\
p<0.001 \\
\mathrm{a}>\mathrm{d}, \mathrm{e}\end{array}$ & $\begin{array}{l}F=2.104 \\
p=0.078\end{array}$ \\
\hline \multirow{6}{*}{ Job } & $\begin{array}{l}\text { Professional ( } \\
\text { a) }\end{array}$ & $364(12.4)$ & $19.03 \pm 5.62$ & $14.01 \pm 3.15$ & $10.82 \pm 2.92$ & $11.54 \pm 3.03$ \\
\hline & $\begin{array}{l}\text { Office work (b } \\
\text { ) }\end{array}$ & $654(22.3)$ & $19.53 \pm 5.19$ & $14.28 \pm 2.76$ & $10.94 \pm 2.64$ & $11.63 \pm 2.75$ \\
\hline & Production (c) & $535(18.2)$ & $20.28 \pm 5.36$ & $13.18 \pm 3.13$ & $11.17 \pm 2.81$ & $12.08 \pm 2.91$ \\
\hline & Student (d) & $384(13.1)$ & $20.43 \pm 5.21$ & $14.46 \pm 2.58$ & $11.26 \pm 2.83$ & $11.45 \pm 2.76$ \\
\hline & Others (e) & 995(34.0) & $19.52 \pm 5.41$ & $13.31 \pm 3.27$ & $10.73 \pm 2.54$ & $12.16 \pm 3.08$ \\
\hline & $\begin{array}{l}\text { F/p value } \\
\text { Post-hoc } \\
\text { (Bonferonni) }\end{array}$ & & $\begin{array}{c}F=5.183 \\
p<0.001 \\
\mathrm{c}, \mathrm{d}>\mathrm{a}, \mathrm{d}>\mathrm{e}\end{array}$ & $\begin{array}{c}F=20.895 \\
p<0.001 \\
\mathrm{a}, \mathrm{b}, \mathrm{d}>\mathrm{c}, \mathrm{e}\end{array}$ & $\begin{array}{c}F=3.856 \\
p<0.001 \\
\mathrm{~d}>\mathrm{e}\end{array}$ & $\begin{array}{c}F=7.192 \\
p<0.001 \\
\mathrm{e}>\mathrm{a}, \mathrm{b}, \mathrm{d} \\
\mathrm{c}>\mathrm{d}\end{array}$ \\
\hline \multirow{5}{*}{$\begin{array}{l}\text { Marital } \\
\text { status }\end{array}$} & Unmarried (a) & 941(32.1) & $20.46 \pm 5.34$ & $14.24 \pm 2.79$ & $10.71 \pm 2.65$ & $11.44 \pm 2.78$ \\
\hline & Married (b) & $1717(58.6)$ & $19.32 \pm 5.24$ & $13.58 \pm 3.10$ & $11.20 \pm 2.75$ & $12.04 \pm 2.94$ \\
\hline & Divorced (c) & $95(3.2)$ & $20.55 \pm 6.25$ & $13.55 \pm 3.33$ & $11.24 \pm 3.08$ & $12.14 \pm 2.97$ \\
\hline & Bereaved (d) & $179(6.1)$ & $19.29 \pm 5.75$ & $12.72 \pm 3.66$ & $11.21 \pm 3.12$ & $12.16 \pm 3.55$ \\
\hline & $\begin{array}{l}\text { F/p value } \\
\text { Post-hoc } \\
\text { (Bonferonni) }\end{array}$ & & $\begin{array}{c}F=10.357 \\
p<0.001 \\
\mathrm{a} \triangleright \mathrm{b}, \mathrm{d}\end{array}$ & $\begin{array}{c}F=16.720 \\
p<0.001 \\
\mathrm{a}>\mathrm{b}>\mathrm{d}\end{array}$ & $\begin{array}{c}F=6.686 \\
p<0.001 \\
\mathrm{~b}>\mathrm{a}\end{array}$ & $\begin{array}{c}F=9.636 \\
p<0.001 \\
\mathrm{~b}, \mathrm{~d} \gg \mathrm{a}\end{array}$ \\
\hline \multirow{7}{*}{ Income } & Low (a) & 629(21.4) & $20.27 \pm 5.51$ & $13.22 \pm 3.27$ & $11.32 \pm 2.91$ & $11.91 \pm 3.14$ \\
\hline & $\begin{array}{l}\text { Medium-low ( } \\
\text { b) }\end{array}$ & 703(24.1) & $19.70 \pm 5.24$ & $13.59 \pm 3.11$ & $10.92 \pm 2.76$ & $11.67 \pm 2.65$ \\
\hline & Medium (c) & $622(21.2)$ & $19.85 \pm 5.39$ & $13.76 \pm 2.96$ & $10.92 \pm 2.78$ & $11.65 \pm 2.96$ \\
\hline & $\begin{array}{l}\text { Medium-high } \\
\text { (d) }\end{array}$ & $480(16.4)$ & $19.50 \pm 5.29$ & $13.87 \pm 3.05$ & $11.11 \pm 2.78$ & $12.00 \pm 2.85$ \\
\hline & High (e) & 271(9.2) & $19.13 \pm 5.39$ & $14.27 \pm 2.89$ & $11.07 \pm 2.51$ & $12.04 \pm 2.85$ \\
\hline & Very high (f) & $227(7.7)$ & $19.08 \pm 5.30$ & $14.89 \pm 2.60$ & $10.83 \pm 2.53$ & $12.34 \pm 2.88$ \\
\hline & $\begin{array}{l}\text { F/p value } \\
\text { Post-hoc } \\
\text { (Bonferonni) }\end{array}$ & & $\begin{array}{c}F=2.883 \\
p=0.013 \\
\text { a >e }\end{array}$ & $\begin{array}{c}F=12.093 \\
p<0.001 \\
\mathrm{f}>\mathrm{a}, \mathrm{b}, \mathrm{c}, \mathrm{d} \\
\mathrm{c}, \mathrm{d}, \mathrm{e}>\mathrm{a}, \mathrm{e}>\mathrm{b}\end{array}$ & $\begin{array}{l}F=2.123 \\
P=0.060\end{array}$ & $\begin{array}{c}F=2.937 \\
p=0.012 \\
\quad \mathrm{f}>\mathrm{b}, \mathrm{c}\end{array}$ \\
\hline \multirow{3}{*}{ Religion } & Yes & $1690(57.7)$ & $20.72 \pm 5.25$ & $13.70 \pm 3.03$ & $11.08 \pm 2.78$ & $11.67 \pm 2.84$ \\
\hline & No & $1242(42.3)$ & $18.99 \pm 5.34$ & $13.77 \pm 3.11$ & $11.02 \pm 2.76$ & $12.00 \pm 3.01$ \\
\hline & $t / p$ value & & $\begin{array}{l}t=8.765 \\
p<0.001 \\
\end{array}$ & $\begin{array}{r}t=-0.646 \\
p=0.518 \\
\end{array}$ & $\begin{array}{c}t=0.584 \\
p=0.559 \\
\end{array}$ & $\begin{array}{r}t=-3.025 \\
p=0.003 \\
\end{array}$ \\
\hline
\end{tabular}

Table 4. Comparisons K-SCS, GARS, and PHQ-15 among groups according to the duration of suicidal ideation

\begin{tabular}{|c|c|c|c|c|c|c|}
\hline & Variable & $\begin{array}{l}\text { Suicidal } \\
\text { ideation } \\
\text { :none (a) } \\
(\mathrm{n}=2,124)\end{array}$ & $\begin{array}{c}\text { Suicidal } \\
\text { ideation } \\
\text { : more than } \\
1 \text { year (b) } \\
(\mathrm{n}=530) \\
\end{array}$ & $\begin{array}{c}\text { Suicidal } \\
\text { ideation } \\
\text { : within } \\
1 \text { year (c) } \\
\text { (n=278) }\end{array}$ & $F / p$ value & $\begin{array}{c}\text { Post-hoc } \\
\text { (Bonferon } \\
\text { ni) }\end{array}$ \\
\hline \multirow{7}{*}{ K-SCS } & Self-kindness & $\begin{array}{c}14.64 \pm 4.0 \\
7\end{array}$ & $\begin{array}{c}14.30 \pm 4.0 \\
9\end{array}$ & $\begin{array}{c}13.21 \pm 3.8 \\
4\end{array}$ & $\begin{aligned} F & =15.780 \\
p & <0.001\end{aligned}$ & $\mathrm{a}, \mathrm{b}>\mathrm{c}$ \\
\hline & Self-judgement & $\begin{array}{c}18.99 \pm 3.7 \\
5\end{array}$ & $\begin{array}{c}17.53 \pm 4.1 \\
3\end{array}$ & $\begin{array}{c}15.68 \pm 4.1 \\
8\end{array}$ & $\begin{array}{c}F=107.5 \\
29 \\
p<0.001\end{array}$ & $a>b>c$ \\
\hline & $\begin{array}{l}\text { Common-humanit } \\
\mathrm{y}\end{array}$ & $\begin{array}{c}11.45 \pm 3.2 \\
6\end{array}$ & $\begin{array}{c}11.96 \pm 3.2 \\
5\end{array}$ & $\begin{array}{c}11.27 \pm 3.1 \\
5\end{array}$ & $\begin{array}{l}F=6.357 \\
p<0.01\end{array}$ & $\mathrm{~b}>\mathrm{a}, \mathrm{c}$ \\
\hline & Isolation & $\begin{array}{c}15.15 \pm 3.2 \\
4\end{array}$ & $\begin{array}{c}14.07 \pm 3.5 \\
4\end{array}$ & $\begin{array}{c}12.50 \pm 3.6 \\
6\end{array}$ & $\begin{aligned} F= & 88.864 \\
p & <0.001\end{aligned}$ & $a>b>c$ \\
\hline & Mindfulness & $\begin{array}{c}12.15 \pm 3.4 \\
6\end{array}$ & $\begin{array}{c}12.23 \pm 3.5 \\
4\end{array}$ & $\begin{array}{c}11.14 \pm 3.2 \\
2\end{array}$ & $\begin{aligned} F & =11.231 \\
p & <0.001\end{aligned}$ & $a>c, b>c$ \\
\hline & $\begin{array}{l}\text { Over-identificatio } \\
\mathrm{n}\end{array}$ & $\begin{array}{c}14.52 \pm 3.0 \\
9\end{array}$ & $\begin{array}{c}12.99 \pm 3.4 \\
7\end{array}$ & $\begin{array}{c}11.74 \pm 3.6 \\
2\end{array}$ & $\begin{array}{c}F=121.5 \\
68 \\
p<0.001\end{array}$ & $a>b>c$ \\
\hline & $\begin{array}{l}\text { Sum of self-comp } \\
\text { assion }\end{array}$ & $\begin{array}{c}86.90 \pm 1 \\
2.16\end{array}$ & $\begin{array}{c}83.08 \pm 1 \\
3.85\end{array}$ & $\begin{array}{c}75.54 \pm 1 \\
4.83\end{array}$ & $\begin{array}{c}F=105.8 \\
67 \\
p<0.001\end{array}$ & $a>b>c$ \\
\hline GARS & & $\begin{array}{c}23.13 \pm 1 \\
3.74\end{array}$ & $\begin{array}{c}30.01 \pm 1 \\
4.23\end{array}$ & $\begin{array}{c}35.37 \pm 1 \\
3.85\end{array}$ & $\begin{array}{c}F=129.8 \\
43 \\
p<0.001\end{array}$ & $c>a>b$ \\
\hline PHQ-15 & & $\begin{array}{c}4.98 \pm 4.5 \\
5\end{array}$ & $\begin{array}{c}7.30 \pm 5.2 \\
8\end{array}$ & $\begin{array}{c}9.79 \pm 6.4 \\
8\end{array}$ & $\begin{array}{c}F=146.9 \\
37 \\
p<0.001\end{array}$ & $c>a>b$ \\
\hline
\end{tabular}

